\title{
L'EMPLOI EXCLAMATIF DE A QUEL POINT
}

\section{Sonia BOUCHAREB}

Université de Sousse, Tunisie

bouchareb.sonia@yahoo.fr

RÉSUMÉ. La locution adverbiale à quel point appartient à une sous-classe particulière d'adverbes appelés adverbes de quantité, tout comme l'adverbe combien, qui sert souvent à la gloser. Cette classification établie à partir du trait sémantique fondamental de degré, permet de présenter les propriétés générales communes aux adverbes de quantité mais non d'en saisir les particularités. L'objectif de ce présent travail est donc d'élaborer une description minutieuse centrée sur à quel point (emploi exclamatif) afin de saisir les spécificités syntaxiques, distributionnelles et sémantiques de ce marqueur exclamatif. Pour ce faire, nous avons procédé par induction en rassemblant un corpus attesté, en français contemporain, représentatif des différents emplois exclamatifs de à quel point. L'observation des données rassemblées nous a permis, en autres, de montrer que, bien qu'appartenant à une même classe grammaticale et sémantique (adverbe indiquant le degré), à quel point et combien ont des fonctionnements syntaxiques différents et des sens en emploi fort singuliers.

Mots-clés : adverbe, conformité, degré, évaluation, intensité, quantité, seuil.

\begin{abstract}
It is commonly acknowledged that the adverbial locution à quel point belongs to a particular sub-class of adverbs called quantity adverbs, as the adverb combien, which serves to replace it. This classification, established from the fundamental semantic trait of degree, allows us to display its common general properties to the quantity adverbs but not to fathom its specificities. The aim of the present study is to set up a minute description centered on à quel point (in its exclamatory use) to seize the syntactic, distributional and semantic specificities relative to this marker. In order to achieve this goal, we have proceeded by induction, i.e. by gathering a certified corpus of different exclamatory uses of à quel point in contemporary French. The analysis of the gathered data has allowed us to show that, while belonging to an identical grammatical and semantic class (an adverb indicating the degree), à quel point and combien have different syntactic functioning and very particular meanings in use.
\end{abstract}

Keywords: adverb, conformity, degree, evaluation, intensity, quantity, threshold. 


\section{INTRODUCTION}

La locution adverbiale à quel point est susceptible de deux emplois différents, selon le type de phrase qui l'englobe :

- un emploi exclamatif dans le cadre d'une exclamation directe ou indirecte :

- A quel point je ne regrette rien (Maniscalco, 2008, p.50)

- J'aimerais tant lui dire à quel point j'aurais été heureuse qu'elle me connaisse ne fût-ce qu'un peu. (Maniscalco, 2008, p.50)

- un emploi interrogatif dans le cadre d'une interrogation directe ou indirecte :

- 3-A quel point vous l'aimez ? (Milner, 1978, p. 260)

- Je me demande à quel point vous l'aimez. (Milner, 1978, p. 260)

Ce présent travail est consacré à l'emploi exclamatif de à quel point. Dans un premier temps, nous nous intéresserons au statut de ce marqueur exclamatif dans ses deux emplois direct et indirect puis nous vérifierons, en nous référant à l'étude de Molinier et Levrier (2000, p. 190) s'il se comporte comme un adverbe de quantité, sous-classe d'adverbes à laquelle il appartient. Cette sous-classe est établie, notamment, à partir du trait sémantique de degré.

Nous nous intéresserons, dans un deuxième temps, au fonctionnement syntaxique de à quel point afin de relever ses spécificités syntaxiques et distributionnelles, en comparaison avec l'adverbe exclamatif combien qui sert souvent à le gloser.

Dans un troisième temps, nous essaierons de relever les différents sens en emploi de cette expression : les valeurs sémantiques attribuables à à quel point sont, nous le verrons, des effets de sens qui dépendent, entre autres, du sémantisme des éléments modifiés par ce marqueur, notamment $\mathrm{du}$ critère gradable /non gradable.

Le cadre théorique adopté est le distributionnalisme, théorie fondée par Bloomfield (1933) et développée par Harris (1951). Cette théorie écarte le sens, c'est-àdire la référence aux objets de la réalité et à la conceptualisation du monde, de l'analyse distributionnelle. Elle permet de segmenter les énoncés, en éléments discrets et la phrase, en constituants immédiats. Selon cette approche, la langue est conçue « comme un ensemble de "discours », de combinaisons construites selon un principe distributionnel » (Leeman, 1999, p. 117). La distribution d'un élément se définit par la somme des tous les environnements possibles dans lesquels il trouve place. Ce principe permet, moyennant l'opération de commutation (substitution d'éléments dans un même contexte), de relever les propriétés distributionnelles des éléments de la langue et de les ranger en classes distributionnelles, lesquelles regroupent des «mots pouvant occuper les mêmes positions dans une structure phrastique » (Riegel et al. 1993, p. 228). Cette méthode consiste alors à " décrire les éléments d'une langue par leur aptitude (possibilité ou impossibilité) à s'associer entre eux pour aboutir à la description totale d'un état de langue en synchronie » (Dubois, 1973, p. 165). Pour ce faire, elle se fonde essentiellement sur un ensemble d'énoncés, réellement produits par le locuteur, appelé corpus.

Il s'ensuit donc que, pour saisir le fonctionnement d'une entité linguistique, notamment à quel point (emploi exclamatif), il faut partir de l'observation d'un ensemble d'énoncés effectivement produits dans une communauté linguistique, constituant un corpus. L'observation de notre corpus ainsi que les différentes manipulations effectuées nous permettra, entre autres, de déduire les différentes distributions et les propriétés syntaxiques de à quel point. Ces différentes conjectures constitueront la base de notre étude sémantique. Par exemple, nous verrons que l'étude des distributions de à quel point, permet, d'une part, de préciser son identité sémantique et d'autre part, d'éclaircir ses conditions d'emploi.

\section{MÉTHODE}

La démarche empirique adoptée consiste donc à partir de l'observation de la forme linguistique à quel point, dans le cadre d'un corpus, échantillon représentatif et homogène de la langue, pour, d'une part, en déduire ses propriétés syntaxiques et ses contraintes distributionnelles et, d'autre part, 
émettre des hypothèses d'ordre sémantique. La méthode suivie n'est autre que la méthode inductive prônée par le distributionnalisme. En effet, la forme étant indissociable du sens, " travailler sur les formes suppose nécessairement la mise en corrélation des observations avec le sens, et de même l'analyse sémantique passe par les formes qui véhiculent les sens que l'on prétend étudier » (Leeman, 2001, p.11).

Cette méthode se base, comme nous l'avons déjà noté, sur la constitution et l'exploitation d'un corpus représentatif des différents emplois exclamatifs de à quel point. Pour ce faire, nous avons eu recours à des exemples attestés relevant du français contemporain. Ces exemples proviennent majoritairement de textes littéraires issus de Frantext, base textuelle sur internet (http://frantext.fr), parmi lesquels Du Bos (1925), Farrère (1907), Adam (1902 ), Bloy (1900), Bourget (1923), Benoît (1919),Samain (1914), Gaulle (1970), Proust (1922) et Simonin (1953) et de Google livres, moteur de recherche accessible sur internet permettant d'accéder à une bibliothèque numérisée, parmi lesquels Maniscalco (2008), Léautaud (1986), Arnold (2009), Lafargue (2012), Simard (2004), Le Thuaut (2011), Natter (2008), Roigoon (2011), Prêtre (1998), Tellez (2008), Salvayre (1995), Gayet et Bonin (2010), Kuttel (1984), Augiéras (2000), Vivas (2006), Lalo (1933), Amadis (2010), Gaffiot (1989), Douzet (2011), Marois (2006), Cohen (1976), Henry (2002),Paquis (2012), LePetit (2013), Paul (2008), Ebeling (1997), Arnaud (2004), Francesco Doni (2004), Netchvolodow (1924), Lacan et Miller (1991), Montgomery (2008). Nous avons également eu recours à des exemples puisés dans certains ouvrages de référence, notamment Milner (1978), Muller (1996), Grevisse (1986, 1993) et Gérard (1980).

\section{RÉSULTATS ET DISCUSSION}

\section{Fonctionnement syntaxique de à quel point}

La locution adverbiale à quel point est présentée par les dictionnaires de langue, notamment le Trésor de la Langue Française (désormais TLF), comme synonyme de l'adverbe combien. Ils peuvent tous deux introduire aussi bien des énoncés directs que indirects. Nous nous proposons, dans le travail qui suit, d'identifier, tout d'abord, le statut de ces marqueurs en emploi exclamatif indirect puis, de présenter un aperçu des points communs et des divergences qui existent entre eux afin de mettre en avant les particularités syntaxiques et distributionnelles de à quel point.

\section{A quel point, adverbe exclamatif}

Comme nous venons de le souligner ci-dessus, à quel point est une locution adverbiale exclamative, qui, comme tous les autres mots exclamatifs, à l'exception de que, peut apparaître en tête d'une exclamative directe ou d'une subordonnée exclamative indirecte :

(5) Seigneur ! à quel point elle a vieilli ! (Léautaud, 1986, p. 1937)

(6) Dans notre chambre, je sentis à quel point mes appréhensions avaient été absurdes. (Arnold, 2009, p. 24)

Les exclamatives indirectes sont, selon Muller, «soit du discours indirect, des exclamations rapportées, soit des actes indirects, après un impératif par exemple » (1996 : 64) :

(7) Regarde à quel point tu es désormais récompensée. (Lafargue, 2012, p. 33)

En emploi exclamatif direct, à quel point est un simple marqueur exclamatif qui a pour rôle de souligner le caractère exclamatif de la phrase. En emploi exclamatif indirect, il est un mot subordonnant qui a pour rôles de marquer la frontière entre la principale et la subordonnée (rôle démarcatif) et d'indiquer le caractère subordonné de la proposition qu'il introduit (rôle subordonnant).

De même que les adverbes interrogatifs (où, quand, pourquoi, comment et combien) qui conservent leur statut adverbial dans une interrogation indirecte (Wagner et Pinchon, 1962, p.549; Riegel, Pellat, et Rioul, 1994, p.839), la locution adverbiale à quel point demeure adverbe exclamatif en emploi indirect, comme combien, mot exclamatif. En effet, le transfert d'un énoncé exclamatif ou interrogatif du discours direct au discours indirect ne change pas la classe grammaticale d'origine $\mathrm{du}$ marqueur exclamatif ou interrogatif. A quel point est adverbe de par sa 
nature et subordonnant de par son rôle. Muller l'identifie d'ailleurs comme « adverbe composé portant sur le degré » (1996: 235) dans l'exclamative indirecte suivante :

(8) Regarde à quel point c'est beau !

Milner note, à ce propos que, la proposition exclamative indépendante et la proposition subordonnée exclamative indirecte, complément d'objet direct du verbe de la principale, ont « exactement les mêmes propriétés morphologiques et sémantiques : la seule différence est que l'une est indépendante et l'autre subordonnée » (1978, p.260).

Quel que soit son emploi (direct ou indirect) à quel point est, comme tous les adverbes, invariables :

9) a-*Samuel n'avait pas réalisé à quels points il a vieilli.

b- Samuel n'avait pas réalisé à quel point il a vieilli. (Simard, 2004, p. 448)

10) a-*Dis-moi à quels points elle est belle!

b-Dis-moi à quel point elle est belle, comment s'appelle-t-elle déjà ? (Le Thuaut, 2011, p. 193)

A quel point a une fonction précise au sein de l'exclamative indépendante ou subordonnée : celle de modifieur d'un verbe comme dans (11), d'un adjectif comme dans (12) ou d'un autre adverbe comme dans (13) toutefois, il est à préciser que les exemples en emploi exclamatif indépendant sont peu nombreux. L'observation de notre corpus montre, en effet, que c'est l'emploi indirect qui semble le plus usité :

(11) a- A quel point vous l'aimez! (Milner 1978, p. 260)

b- Je sais à quel point vous l'aimez. (Milner 1978, p. 260)

(12) a- A quel point, loin de toute autorité, de toute direction, la vie devient invivable. (Prêtre, 1998, p. 17)

b- Elise réalise à quel point il est heureux et son bonheur la touche au plus haut point. (Natter, 2008, p. 282)

(13)- a- Et pourtant, l'heure du châtiment fut, comme toujours, l'heure de la miséricorde. Les hommes d'église réalisèrent à quel point, follement, ils avaient poursuivi l'attrait du plaisir et de la puissance. (« benoît et moi », s.d.)
Compte tenu de son sémantisme, à quel point appartient à une sous-classe particulière d'adverbes appelés " adverbes de degré », ou adverbes «de mesure» (Buyssens, 1975, p. 462), ou « intensifs » (Feuillet, 1991, p. 49), ou encore adverbes «de quantité » (Molinier, 1990, p. 37). Cette sous-classe d'adverbes se caractérise aussi par un ensemble de propriétés d'ordre syntaxique et sémantique recensées par Molinier et Levrier (1990) qui leur a permis de dresser une liste de ses principaux constituants : beaucoup/très, autant/aussi, tant/si, plus, moins, davantage, trop, peu, assez, ne...pas du tout (op.cit., p. 190). Nous nous proposons, dans le travail qui suit, de voir si à quel point se comporte comme un adverbe de quantité.

\section{A quel point, une locution adverbiale de quantité?}

Les auteurs de la Grammaire des adverbes, Description des formes en -ment énumèrent cinq propriétés générales communes aux adverbes de quantité :

1re propriété : ils peuvent modifier indifféremment un verbe, un adjectif ou un adverbe. L'observation de notre corpus montre que à quel point peut porter :

- $\quad$ sur un verbe :

(14) a- A quel point j'ai vieilli. Alors que toi, tu es resté le même. («fanfiction », s.d.)

b- Il remarque soudain à quel point elle a vieilli. ( Roigoon, 2011, p. 26)

- $\quad$ sur un adjectif:

(15) a- A quel point il est grand! (Muller 1996, p. 63)

b- Elise réalise à quel point il est heureux et son bonheur la touche au plus haut point. (Natter, 2008, p. 382)

- sur un adverbe, en emploi exclamatif indirect uniquement

(16) Mais, mec, tu ne te rends plus du tout compte à quel point il chante bien, tellement tu l'as entendu chanter ! (ralphgull, s.d.)

2e propriété : les adverbes de quantité ne se prêtent pas à l'extraction en c'est...que :

(17) -a- Imbécile! A quel point tu es dépassé ! Que vient faire ta mère làdedans? (Tellez, 2008, p. 17) b-* Imbécile! C'est à quel point que tu es dépassé ! 
3e propriété : ils peuvent fonctionner comme déterminant indéfini du substantif. Ils sont alors suivis de la préposition de. A quel point est incompatible avec un substantif, contrairement à combien :

(18) a- Oh! Combien de marins, combien de capitaines/ [...]/ Dans ce morne horizon se sont

évanouis! (Hugo, cit. Grevisse et Goosse 1993, p. 616)

b-* Oh! A quel point de marins, à quel point de capitaines/ [...]/ Dans ce morne horizon se sont évanouis !

C'est aussi le cas des adverbes de quantité si, très et aussi mais cela ne remet pas en question leur appartenance à la sous-classe des adverbes de quantité.

$\underline{4}$ eropriété : ils ne peuvent que figurer dans le voisinage immédiat du verbe, à droite du verbe quand il s'agit d'une forme verbale simple, à droite de l'auxiliaire quand il s'agit d'une forme verbale composée. Il est à noter que cette propriété ne s'applique pas à à quel point car, en tant qu'adverbe exclamatif, il figure toujours en tête de la phrase exclamative indépendante ou subordonnée :

(19) a- Tu sais à quel point j'aime les roses! (doucetoile, s.d.)

b- *Tu sais j'aime à quel point les roses!

c- ${ }^{*}$ Tu sais j'ai à quel point aimé les roses!

5e propriété : la cinquième propriété mentionnée est une propriété interprétative : en contexte verbal, les adverbes de quantité ont soit une interprétation de type quantitatif, soit une interprétation de type intensif. C'est notamment le cas de à quel point qui oriente l'interprétation de l'énoncé exclamatif vers une valeur élevée soit dans l'ordre de la qualité, soit dans l'ordre de la quantité :

(20) Je hais mon père, au-delà de toute mesure, personne, monsieur Jean, ne peut imaginer à quel point je le hais. (Salvayre, 1995, p. 86)

L'expression à quel point je le hais peut être paraphrasée par «je le hais beaucoup = énormément ", c'est la quantité de haine qui est évaluée ou par « je le hais profondément = de manière intense ", c'est l'intensité de la haine qui est alors évaluée (sa haine est très profonde).
Comme nous venons de le voir, à quel point vérifie les propriétés générales des adverbes de quantité même s'il n'apparaît pas dans la liste mentionnée par Molinier et Levrier. Comparer le comportement syntaxique et les distributions de combien et à quel point nous permettra de mieux saisir les spécificités de cette locution adverbiale exclamative.

\section{A quel point/combien: convergences et divergences}

Les adverbes exclamatifs combien et $\grave{a}$ quel point peuvent tous deux porter sur des éléments gradables. Il peut s'agir:

- d'un verbe :

(21) a- Mais, combien je regrette notre poste à Stockholm ! (Kuttel, 1984, p. 46)

$a^{\prime}-{ }^{*}$ Combien la pluie cesse!

b- Et Dieu sait combien je regrette sa mort. (Vivas, 2006, p. 362)

$b^{\prime}$-*Je sais combien la pluie cesse.

(22) a- A quel point j'ai souffert de la solitude! (Augiéras, 2000, p. 116) $\mathrm{a}^{\prime}-{ }^{*}$ A quel point la pluie cesse ! b- « Je suis un grand malade, besoin de repos, laissez-moi récupérer, vous ne voyez pas à quel point j'ai souffert ». (Gayet et Bonin, 2010, p. 71)

$\mathrm{b}^{\prime}$ - ${ }^{*} \mathrm{Je}$ sais à quel point la pluie cesse.

- d'un adjectif :

(23) a- Combien il est grand! (Milner, $1978: 265)$

$a^{\prime}-*$ Combien la table est carrée !

b- Je sais combien est grand le nouveau sacrifice que je te demande, et combien il t'en coûtera de te l'imposer ; mais ma confiance en ton amitié me fait tout oser. (Lalo, 1933, p. 240)

$\mathrm{b}^{\prime}$ - *J'admire combien la table est carrée.

(24) a- ? A quel point elle est belle ! (exemple forgé)

$a^{\prime}-{ }^{*}$ A quel point la table est carrée ! b- S'avançant vers son cocon, il ne put que constater à quel point elle était belle, ne se

privant pas de l'admirer. Amadis, 2010, p. 216)

$\mathrm{b}^{\prime}$-*Vois à quel point la table est carrée. 
- d'un autre adverbe: il est à noter, néanmoins, que l'exclamation direct en à quel point portant sur un adverbe est d'une acceptabilité assez douteuse. Nous ne trouvons aucun exemple, dans notre corpus, attestant un tel emploi en indépendante.

(25) a-Combien profondément, combien passionnément, combien tendrement, je vous aimais et vous croyais. (Gaffiot, 1989, p. 84)

$a^{\prime}-{ }^{*}$ Combien il parle rapidement

b- Vous savez combien passionnément j'ai cherché dans les œuvres d'art l'âme de l'artiste pour les comprendre (...) (BernardGriffiths, 1998, p. 95)

$\mathrm{b}^{\prime}$-*Je sais combien tu parles $^{*}$ rapidement.

(26) a- ?A quel point il mange bien ! (exemple forgé, acceptation douteuse)

$a^{\prime}-* A$ quel point il mange goulûment !

b- Souvent, cette création incroyable prend la forme d'une photo de nourriture, l'internaute s'empressant de partager son plat tout juste sorti du four pour montrer à tous ces contacts à quel point il mange bien. («jbastardidaumont ", s.d.)

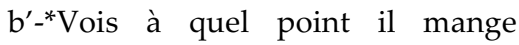
goulûment.

Bien qu'il soit généralement admis que les verbes, les adjectifs et les adverbes acceptant la modification par un adverbe de quantité, notamment à quel point et combien, sont gradables, l'observation de notre corpus montre que ces deux adverbes exclamatifs peuvent aussi porter sur certains verbes ou adjectifs n'admettant pas le degré, dans ce cas, ces adverbes ont un sens différent:

(27) a- Et comme il avait raison, à quel point je le sais aujourd'hui !

(Du Bos, 1925, p. 348)

b- Oh combien je le sais.

(Douzet, 2011, p. 38)

(28) a- C'est là qu'il a compris à quel point il est français.

(Marois , 2006, p. 104)

b- Frédéric Henri est le plus tolérant, le plus élégant et le plus souriant de tous les princes, ce qui ne l'empêche pas d'être fort; et combien il est Français avec son entourage (...)

(Cohen, 1976, p. 424)

Milner (1978, p. 270) précise que certains adjectifs, notamment les adjectifs désignant des nationalités, changent de sens suivant qu'ils admettent le degré ou non. Il oppose ainsi il est français «il est de nationalité française » et il est très français «il a le caractère d'un Français ». Dans l'exemple (28), seul le second sens est possible.

$C^{\prime}$ est aussi le cas de certains verbes. Ils expriment alors non pas le degré de quantité ou d'intensité mais le degré de conformité à la réalité comme dans (27) (Il le sait vraiment). Ils peuvent également exprimer la durée (compatible avec un complément de temps indiquant la durée comme longtemps, durant plusieurs jours, etc.) ou la répétition d'une action ou d'un processus (compatible avec souvent) comme dans (29) :

(29) a- [...] vous n'imaginez pas à quel point ces vieilles maisons turques gémissent et tremblent sous les rafales.

(Farrère, 1907, p. 111-112)

b- Apparemment j'irai bientôt dormir au sein de notre mère la terre, en me transformant par les mille vies de la corruption...écoute, petit, écoute...vois combien ma vieillesse implore.

(Adam, 1902, p. 110)

Dans le cas de combien, l'adjectif peut être attribut comme dans (30) ou épithète inclut dans un groupe nominal (désormais (GN)) comme dans (31), contrairement à quel point qui ne peut porter que sur un adjectif attribut, le verbe attributif étant être ou devenir, selon Bacha (2000 : 201) :

(30) a- O combien je suis heureux d'avoir pu vivre en directe de tels événements! (Henry, 2002, p. 314) b- A quel point je suis seul ! (Paquis, 2012, p. 65)

a'- Voyez combien je suis devenu vieux. (exemple forgé)

$b^{\prime}$ - Elle est peut être en ce moment en train de dire à Eric combien je l'ai déçue, à quel point je suis devenue moche alors qu'elle avait le souvenir d'un petit garçon 
bourré de charme. (LePetit, 2013, p. 92)

(31) a- Combien vous avez de grandes dents! (cit. Milner 1978, p. 265)

b- *A quel point vous avez de grandes dents!

Combien peut jouer, à lui seul, le rôle de sujet, contrairement à à quel point :

(32) a- Combien voudraient être à votre place! (Grevisse 1986, p. 662)

b- *A quel point voudraient être à votre place!

De même combien, à la différence de à quel point, peut modifier un nom appartenant à un syntagme prépositionnel, il suit alors la préposition :

(33) a- Avec combien de joie on y trahit sa foi! (Racine (H. 142) cit. Gérard 1980, p. 29)

$\mathrm{b}$ - * Avec à quel point de joie on $\mathrm{y}$ trahit sa foi !

Comme combien, à quel point peut être immédiatement suivi par le terme gradable support de l'exclamation (emploi indirect uniquement), il s'agit néanmoins, dans les deux cas, d'une construction assez rare :

(34) a- Combien facilement la vie se reforme, se referme (Gide, cit. Grevisse 1986, p. 661)

$\mathrm{b}-{ }^{*}$ A quel point grand il est!

$a^{\prime}$ - « Combien belle est la forme de son (de Sarah) visage, et combien adorable et douce la chevelure de sa tête ! [...]».

(Paul, 2008, p. 125)

$b^{\prime}$ - Quelle meilleure façon de montrer votre tout-petit et d'autres permettent de voir à quel point beau, il est. (« esty »,s.d.)

Combien et à quel point peuvent constituer, à eux seuls, une phrase mais seul combien peut apparaître dans une construction absolue, c'est-à-dire une construction sans verbe (cf. (ex. (36)) :

(35) a- Combien!

(Bloy, 1900, p. 333)

b- A quel point !

(Bourget, 1923, p. 10)

(36) a- Le joli système d'éducation et combien pratique! (Prévost $(\mathrm{H}$. 141) cit. Gérard 1980, p. 31)

b- * Le joli système d'éducation et à quel point pratique!

L'observation $\mathrm{du}$ corpus montre également que les exclamatives en combien et à quel point permettent l'inversion du sujet nominal et pronominal. Dans le cas de à quel point, nous remarquons que, lorsque le sujet est nominal, les deux types d'inversion sont possibles, en emploi indirect, et inexistants en emploi direct :

(37) a- Mais voyez à quel point $m$ 'intéressent vos révélations : je diffère cette question d'ordre privé. ( inversion simple) (Benoît, 1919, p. 149)

b- Cette notion de l'arbitraire, à quel point, des années durant, ne m'a-t-elle pas tyrannisé ! (inversion complexe)

(Du Bos, 1925, p.246)

Dans le cas de combien, seule l'inversion simple du sujet nominal est possible dans les exclamatives directes et indirectes, voire même, selon Bacha (2000), obligatoire lorsque cet adverbe exclamatif porte sur un adjectif attribut antéposé :

(38) a- Combien m'a fait plaisir votre lettre! (cit. Bacha 2000, p.69) Vs

Combien votre lettre m'a-t-elle fait plaisir! (Idem)

b- Je sais trop combien vaine est la rébellion. (Samain, 1914, p. 160)

Lorsque le sujet est pronominal (pronom personnel ou ce, on), l'inversion est obligatoire lorsque la phrase exclamative en $\grave{a}$ quel point ou combien comporte une négation oratoire, équivalente sémantiquement à une affirmation (Grevisse et Goosse 1994, §620). Elle semble aussi possible dans une exclamative affirmative même si cette construction est jugée «peu acceptable » par Bacha (Idem.):

(39) a- Combien n'ai-je pas écrit de récits à cette époque! (Green, cit. Grevisse 1986, p. 662)

b- Combien souvent ai-je été tenté ! C'est un miracle de Dieu que je n'aie pas chuté. Combien souvent suis-je tombé ! C'est un miracle de Dieu que j'aie reçu son pardon. ( Ebeling, 1997, p. 154)

$a^{\prime}$ - A quel point n'avais-je pas tort. (Du Bos, 1927, p. 284)

$\mathrm{b}^{\prime}$ - Mais à quel point le suis-je aujourd'hui! Gaulle (de), 1970, p. 282) 
Alors que, selon Bacha, l'inversion du sujet nominal est obligatoire lorsque combien porte sur un adjectif attribut antéposé, nous notons qu'elle est facultative dans le cas de à quel point :

(40) a- Combien importante fut cette décision ! (cit. Bacha 2000, p. 69)

b- (...) il était incroyable à quel point sa vie était successive, et fugitifs ses plus grands désirs. (Proust, 1922, p. 387)

Contrairement à à quel point, combien est susceptible de quantifier un nom :

(41) a- Oh, combien de coquins il y a là, qui seraient mieux sous terre que dessus !

(Francesco Doni, 2004, p. 111)

$\mathrm{b}$ - * Oh, à quel point de coquins il y a là, qui seraient mieux sous terre que dessus!

Les exemples relevés comportant à quel point $+\mathrm{de}+N$ montrent qu'il s'agit d'une combinaison libre équivalente à à quel degré/niveau la commutation par combien étant alors impossible

(42) a- Les indications ci-dessus montrent suffisamment à quel point de/ ${ }^{*}$ combien de perfection les Juifs ont poussé leur organisation (...)

(Netchvolodow, 1924, p. 127)

b- Les indications ci-dessus montrent suffisamment à quel niveau / à quel degré de perfection les Juifs ont poussé leur organisation (...)

Ces différentes remarques montrent que combien et à quel point, bien qu'assez proches dans leur fonctionnement syntaxique et leurs distributions, ont chacun leurs spécificités qui en font des marqueurs exclamatifs à part. Il s'avère aussi, que l'appartenance de ces éléments à un même ensemble, basée sur le trait sémantique de degré, ne permet pas d'en prévoir les propriétés syntaxiques. Gaatone note, à ce sujet que « [...] les comportements des adverbes de degré sont bien trop divergents pour qu'on puisse systématiquement les déduire de l'appartenance à un même ensemble » (2008a : 628).

\section{Sens de à quel point}

L'homogénéité syntaxique de la sousclasse des adverbes de degré que semble lui conférer le trait sémantique de degré est donc illusoire, du moins en ce qui concerne les adverbes combien et à quel point, de même que son semblant d'homogénéité sémantique. En effet, une analyse quelque peu exhaustive des valeurs sémantiques de à quel point permet d'en saisir toute la singularité.

\section{A quel point: quantifieur ou intensifieur?}

Comme les autres marqueurs exclamatifs, à quel point n'exprime pas par lui-même le haut degré la preuve étant, selon Milner (1978, p.260), qu'il puisse apparaître dans des contextes non-exclamatifs (emploi interrogatif). Celui-ci affirme que la nuance intensive présente dans l'exclamative indirecte (43) Je sais à quel point vous l'aimez ne fait pas partie de la locution adverbiale à quel point puisqu'elle n'est pas attestée pour l'interrogative indirecte (44) Je me demande à quel point vous l'aimez.

D'un point de vue compositionnel, à quel point est constitué de la préposition à, du déterminant quel et du nom point qui, en emploi exclamatif, acquièrent une valeur particulière. La préposition à indique, conformément au sens général de «destination» qui lui est généralement attribué, la direction vers un point d'aboutissement, en l'occurrence la ou les valeur (s) de degré extrême à attribuer à l'élément gradable évalué. Le déterminant exclamatif quel, quant à lui, qui précède le nom point lui donne une valeur intensive : il stipule que le point ou degré atteint par l'élément gradable se situe dans la zone des hautes valeurs susceptibles de lui être attribuées, valeur que le locuteur ne peut préciser vu son degré extrême, «degré tel qu'il échappe à l'énonciation », selon Gérard (1980: 3) . Le nom point précise, lui, que le point en question est un espace (physique, intellectuel ou mental) minimal ou plus précisément une zone à déterminer sur cette échelle de valeurs.

Il est généralement admis que lorsque l'adverbe de degré, notamment à quel point, modifie le degré des adjectifs et des adverbes, il dénote l'intensité, lorsqu'il marque le degré du substantif, auquel cas il est automatiquement suivi de la préposition de (cas de combien mais non de à quel point), il 
dénote uniquement la quantité et quand il marque le degré des verbes, il exprime la quantité ou l'intensité, selon le sens du verbe comme en témoignent les exemples suivants :

(45) Il vivait si simplement qu'on ne soupçonnait pas à quel point il était riche, et l'eût-on su qu'on se fût trompé encore, l'ayant cru alors avare, alors que personne ne fut jamais si généreux. (propriété, intensité)

(Proust, 1922, p. 183)

(46) Comme introduction aujourd'hui, je voulais seulement vous dire à quel point profondément nous intéresse ce statut [le statut du maitre], dont il vaut de garder l'énonciation pour un prochain pas. (propriété, intensité) (Lacan et Miller, 1991, p.:22)

(47) (...) je ne me rendais pas compte à quel point il avait vieilli. (état, intensité)

(Proust, 1922, p. 860-861)

(48) Il faut absolument que je parle à quelqu'un qui peut comprendre à quel point je souffre. (quantité, souffrir énormément)

(Montgomery, 2008, p. 174)

La différence entre quantité et intensité, et, par conséquent, entre quantifieur et intensifieur, est, selon Gaatone, induit par «la nature des objets extra linguistiques mesurés »(2008b, p. 2495) telle que états ou propriétés pour les adjectifs et adverbes, entité ou substance pour les noms, états ou actions ou " événements réitérables » (Romero 2001, p.11) pour les verbes.

En tant qu'adverbe de degré, à quel point institue donc une certaine contrainte sur les éléments qu'il modifie. Ceux-ci sont, comme nous venons de le noter ci-dessus, des éléments gradables, c'est-à-dire des éléments dénotant des notions susceptibles de varier en degré. Il s'agit d'adverbes, d'adjectifs, de certains verbes mais aussi de certains substantifs qui ont subi un processus d'adjectivation appelés par Goes « substantifs adjectivés»(1999, p. 150). Ces substantifs, employés sans déterminant en fonction attribut, peuvent être modifiés par un adverbe de degré et accepter la forme comparative ou superlative (Marie est très femme, Sophie est plus femme que Marie, cité par Whittaker (2002, p. 134)
Or, nous avons déjà noté que cette locution adverbiale de degré peut porter sur des éléments non-gradables, ce qui lui confère différentes valeurs sémantiques en emploi, comme nous allons le voir dans le travail qui suit. Il semblerait donc que la classification des éléments susceptibles d'être modifiés par à quel point selon qu'ils admettent ou non le degré ne puisse pas rendre compte de ses différentes interprétations.

\section{Autres valeurs sémantiques de à quel point}

Le cotexte, et plus précisément, le sémantisme de l'élément modifié par le marqueur exclamatif à quel point est un facteur essentiel dans l'identification de sa valeur sémantique. Dans l'exemple

(49) Vous le croiriez pas, docteur, lui a fait Pierrot, à quel point mon ami Maxpeut être gamin et entêté pour son âge! (Simonin, 1953, p. 58)

L'évaluation gamin et entêté est l'objet d'un jugement personnel négatif (celui de Pierrot). À quel point porte sur les adjectifs gradables gamin et entêté et souligne que l'intensité des propriétés auxquelles renvoient ces deux adjectifs est jugée excessive. Le groupe prépositionnel pour son âge confirme et explicite cette idée de seuil dépassé : Max est trop gamin et entêté pour son âge. Il ne s'agit pas, selon Noailly «d'une évaluation sur une échelle qui irait du plus au moins » (1999:58) dans la mesure où Max peut être jugé trop gamin et entêté alors qu'il ne l'est pas du tout ou très peu. L'évaluation de Pierrot peut être sujet à discussion car elle fait état de son système personnel d'estimation relativement à un sujet donné.

Comme nous venons de le voir, la locution adverbiale à quel point exprime l'idée d'une limite atteinte et dépassée et est donc paraphrasable par l'adverbe trop.

A quel point peut aussi dénoter l'idée de fréquence et de répétition du procès exprimé par le verbe, pivot exclamatif comme dans l'exemple :

(50) Ce qui est curieux et ce sur quoi je ne peux m'étendre, c'est à quel point, vers cette époque-là, toutes les personnes qu'aimait Albertine, 
toutes celles qui auraient pu lui faire faire ce qu'elles auraient voulu, demandèrent, implorèrent, j'oserai dire, mendièrent, à défaut de mon amitié, quelques relations avec moi.

(Proust,1922, p. 706)

Les verbes demander, implorer et mendier n'admettent pas le degré: il ne s'agit ni de verbes affectifs ni de verbes indiquant un changement de qualité, verbes susceptibles de varier en degré (Milner 1978) néanmoins, certains de ces verbes peuvent être modifiés par l'adverbe beaucoup qui n'exprime pas l'intensité du processus mais sa répétition, sa fréquence :

51) a- *Elles demandèrent beaucoup une relation avec moi.

vs

$a^{\prime}$ - Elles demandèrent sans cesse une relation avec moi.

b- ? Elles implorèrent beaucoup une relation avec moi.

Vs

$b^{\prime}$ - Elles implorèrent inlassablement une relation avec moi.

c- Elles mendièrent beaucoup
Vs
$c^{\prime}$ - Elles mendièrent souvent.

Les verbes demander, implorer, mendier sur lesquels portent à quel point sont des verbes non-conclusifs (ou imperfectifs) dans la mesure où ils désignent, selon Bacha (2000, p. 19), des procès qui ne comportent pas dans leur signifié l'idée de leur fin ; ils sont donc susceptibles de se prolonger indéfiniment sauf intervention de circonstances extérieurs. Riegel, Pellat, et Rioul, 1994, (1994, p. 294) remarquent que ces verbes sont compatibles avec des adverbes du type longtemps, plusieurs jours... :

(51) d- Les personnes se sont longtemps demandé, ont longtemps imploré, ont longtemps mendié.

Selon Bacha (2000), la compatibilité qui existe entre les verbes non-conclusifs et l'idée de fréquence ou de durée véhiculée par la présence de à quel point s'explique par le fait que ces verbes incluent "les sèmes de fréquence et/ou de durée et/ou d'intensité » (p. 22). Il s'ensuit que ce marqueur exclamatif acquiert, dans cet emploi, une valeur itérative et durative qui en fait un quantifieur dans la mesure où, selon Romero (2001, p. 71), il exprime de la quantité appliquée à des évènements réitérables.

Autre valeur en emploi de à quel point : celle de conformité et d'intensité comme l'illustre l'exemple suivant :

(52) Mais en tout cas, faute du volet Gide, il faut montrer à quel point Gide est un Grec d'aspiration et non de naissance. (Du Bos, 1927, p. 375)

Dans cet exemple, la locution adverbiale à quel point porte sur le terme grec qui désigne une nationalité. Ce terme a deux sens différents : il indique soit la nationalité grecque (sens rejeté par l'énoncé même), soit le caractère grec. Il est vrai que dans le cas de l'adjectif grec, la propriété dénotée par cet adjectif dans son second sens, est gradable (Gide est très grec) et donc compatible avec l'exclamation ce qui n'est pas le cas du nom dans la mesure où il est question d'un nom ordinaire et non d'un nom de qualité. En tant que nom ordinaire, grec définit une classe dont les membres sont reconnaissables à des propriétés objectives communément admises, dites «propriétés typiques » (Kleiber 1999, p. 69). Dire À quel point Gide est un Grec d'aspiration et non de naissance !, phrase dans laquelle le nom grec figure comme attribut du sujet, c'est non seulement affirmer l'appartenance de Gide à la classe des Grecs mais c'est aussi affirmer le haut degré de conformité de Gide à cette même classe. Le nom grec est donc un nom " classifiant », c'est-à-dire un nom qui, lorsqu'il est attribut avec le verbe être, permet à la proposition qui l'englobe d'être interprétée comme « un jugement d'appartenance d'un élément à une classe ou la subsomption d'un objet sous un concept » (Milner 1978, p. 295).

La locution adverbiale à quel point, commutable avec vraiment, souligne que l'évaluation s'effectue en termes de conformité simple ou élevé à la réalité. A cette valeur de conformité peut se greffer une valeur intensive : la conformité à la réalité est telle que l'élément évalué se rapproche énormément $\mathrm{du}$ prototype, meilleur représentant de la classe à laquelle il appartient. 
Il s'avère donc que les différentes valeurs sémantiques de à quel point, relevées dans le cadre de cette présente étude, sont, en fait, des effets de sens qui émanent, entre autres, de son emploi en contexte exclamatif et du cotexte, notamment le sémantisme des éléments modifiés par cette locution adverbiale de degré.

\section{CONCLUSION}

Affirmer l'appartenance de la locution adverbiale à quel point à une sous-classe particulière d'adverbes nous permet certes d'en relever les propriétés communes mais non d'en déduire ses particularités syntaxiques, distributionnelles et interprétatives qui en font un adverbe de degré à part. Comparer le fonctionnement syntaxique de à quel point à combien, adverbe souvent utilisé par les dictionnaires de langue pour la gloser, nous a permis de nous rendre compte qu'il n'existe pas deux mots ayant les mêmes caractéristiques ce qui s'oppose à toute tentative de systématisation et nous ramène à l'idée que, pour une description aussi fidèle que possible de la langue, il faut nécessairement passer par une étude à part de chaque élément du lexique. Seule une étude au cas par cas permet de saisir toute la richesse et la complexité de la langue.

Nous avons donc essayé, dans le cadre de ce présent travail, de présenter une étude syntactico-sémantique assez exhaustive de l'emploi exclamatif de à quel point, étiqueté comme adverbe de degré. Nous avons relevé, via l'observation d'un corpus riche et varié, ses particularités syntaxiques et ses possibilités distributionnelles. Ce travail nous a permis, entre autres, de confirmer l'identité grammaticale et sémantique de cette locution en point.

Nous avons aussi remarqué que, en emploi exclamatif, à quel point véhicule l'idée de haut degré dans l'ordre de la qualité ou de la quantité : il porte alors sur des éléments gradables (adjectifs, adverbes et verbes). Néanmoins, nous avons noté que ce marqueur exclamatif peut aussi exprimer l'idée d'une limite atteinte et dépassée ou l'idée de répétition du procès ou encore l'idée de conformité simple ou élevée à la réalité et ce, lorsqu'il porte sur des éléments non gradables (notamment, adjectifs et verbes). Ces différents sens en emploi sont, dans ce cas de figure, des effets de sens qui émanent de la combinaison de différents facteurs, entre autres, la structure exclamative de la phrase qui englobe à quel point et le sémantisme de l'élément modifié par cette locution adverbiale.

\section{REMERCIEMENTS}

Je remercie Mme Jacqueline BACHA, directrice $\mathrm{du}$ laboratoire de recherche Langues, Discours et Cultures (Institut Supérieur des Sciences Humaines de Jendouba, TUNISIE) pour ses judicieux conseils et ses remarques pertinentes qui $\mathrm{m}^{\prime}$ ont permise d'améliorer ce présent travail. Je remercie également toute personne ayant participé de près ou de loin à l'élaboration de cet article.

\section{RÉFÉRENCES}

Adam, P. (1902). L'enfant d'Austerlitz. Paris : Olledorff

Amadis, E. (2010). Les Rescapés. Paris: Books on Demand France (BoD)

Arnold E. F. (2009). Malgré nous, malgré la Shoah: irrésistiblement, Paris : Editions du Cygne

Augiéras, F. (2000). Lettres à Paul Placet, 19521971. Périgueux : Fanlac

Bacha, J. (2000). L'exclamation, Approche syntaxique et sémantique d'une modalité énonciative. Paris: L'Harmattan

Benoît, P. (1919). L'Atlantide. Paris: Albin Michel

[Benoît et moi] (s.d.) Repéré le 23/09/2017 à http://benoit-et-moi.fr/2015-

II/actualite/un-chatimentmisericordieux.php

Bernard-Griffiths, S. (1998). Variétés sur Michelet. Saint- Genouph : Nizet, Cahier romantique $\mathrm{n}^{\circ} 3$

Bloomfield, L. (1933). Language. New York: Henry Holt

Bloy, L. (1900). Journal 1 : Mon journal : 18961900. Paris : Mercure de France

Bourget, P. (1923). La Geôle. Paris : Pion

Buyssens, E. (1975). La classification des adverbes, Revue roumaine de linguistique, 20, $\mathrm{n}^{\circ}$ 5, 461- 463 
[doucetoile] (s.d.) Repéré le 17/07/2017 à http://douceetoile.over-

blog.com/article-une-petite-boite66705818.html

[fanfiction](s.d.) Repéré le12/03/2016 à https://www.fanfiction.net/s/7052969/1 /Si-près

Douzet, A.L. (2011). Saga La porte, Tome 2, Blanche Tepes. Paris : Magic Tales

Du Bos, Ch. (1925). Journal: t.2 (1924-1925). Paris, Corrêa

(1927). Journal : t .3 (1926- 1927. Paris, Corrêa

Dubois, J. et al. (1973), Dictionnaire de linguistique. Paris, Librairie Larousse

Ebeling, G. (1997). Prédications illégales, Berlin 1939- 1945. Genève : Labor et Fides

[esty] (s.d.) Repéré le 20/09/2017 à https://www.etsy.com/ca-

fr/listing/106449515/gilet-onesiescravate-pour-les-garcons-3

Farrère, Cl.(1907). L'Homme qui assassina. Paris : Ollendorff

Feuillet, J. (1991). Adjectifs et adverbes : essai de classification, Travaux linguistiques du Cerlico 3, Les états de l'adverbe, 3558

Francesco Doni, A. (2004). Humeurs et paradoxes, florilège présenté et traduit par Michel Arnaud, «Paroles d'ailleurs ». Grenoble: ELLUG

Gaatone, D. (2008a). Les adverbes de degré en français: interface sémantique/syntaxe, in Merete Birkelund, Maj-Britt, Mosegaard Hansen, Coco Norén (éds) L'énonciation dans tous ses états. Mélanges offerts à Henning Nølke à l'occasion de ses soixante ans, Bern, Peter Lang, 623-643.

Gaatone, D. (2008b). Un ensemble hétéroclite: les adverbes de degré en français, CD-ROM des actes du Congrès Mondiale de Linguistique Française, Paris, 9-12 juillet 2008, pp.2495-2504- article disponible sur le site :

http://www.linguistiquefrançaise.org

Gaffiot, J-M. (1989), Netchaïef, Lausanne (Suisse), L'Age d'Homme
Gaulle, Ch. (de). (1970). Discours et messages. 1. Pendant la guerre. 1940- 1946. Paris: Pion

Gayet,J-L. et Bonin, M. (2010). Pas si bête! Les psychothérapeutes sont-ils des gens sérieux ? Paris : Société des écrivains

Garagnon, A.M. et Calas, F. (2002). La phrase complexe, De l'analyse logique à l'analyse structurale, Paris, Hachette

Gérard, J. (1980). L'exclamation en français. La syntaxe des phrases et des expressions exclamatives. Tübingen : Niemeyer

Goes, J. (1999). L'adjectif, Entre nom et verbe. Paris, Bruxelles : Duculot

Grevisse M. , Goosse A. (1986). Nouvelle Grammaire française. Paris : Duculot

Grevisse, M. (1993). Le bon usage, 13e édition refondue par A. Goosse. Paris: Duculot

Harris, Z. (1951), Methods in Structural Linguistics. Chicago: University of Chicago Press

Henry, Cl.- A. (2002). Adolescence, cette autre vie. Paris : Publibook

[jbastardidaumont] (s.d.) Repéré le 08/03/2016 à

www. jbastardidaumont.com/2013/05/.../quitterfacebook-facebook-rend-tristel

Kuttel,M. (1984), La Malvivante. Suisse : L'Age d'Homme

Kleiber G. (1990). La sémantique du prototype, Catégories et sens lexical. Paris : PUF

Lacan J. et Miller A. (1991), L'Envers de la psychanalyse : 1969-1970. Paris : Seuil

Lafargue, P. (2012), Chemins d'Eternité, Enseignements et signes de l'Au-delà. Paris : Lanore

Lalo, P. (1933). Richard Wagner ou Nibelung. Paris : Flammarion

Léautaud P. (1986). Journal littéraire; Février 1940- Février 1956. Paris: Mercure de France

Leeman, D. (1999). L'unité lexicale dans la perspective harrissienne, $\operatorname{Linx} 40$, Centre de Recherches Linguistiques de l'Université de Paris X- Nanterre, 117136

Leeman, D. (2001). Quand les formes informent: de la grammaire à la sémantique, Le Français aujourd'hui 135, 11-19 Repéré à http://www.cairn.info/revue-le- 
francais-aujourd-hui-2001-4-page-

11.htm

Léon, B. (1900). Journal 1 : Mon journal : 18961900. Paris : Mercure de France

LePetit, S. (2013). La Korrandine de Tevelune, La Vallée de l'Argentor. Paris: Hélène Jacob

Le Thuaut, L. (2011), Joseph-Marie Coët. Paris : Publibook

Maniscalco, S. (2008), Peau d'âme. LiègeBressoux : Dricot

Marois, A. (2006). Accidents de parcours. Montréal (Québec)) : la courte échelle inc.

Milner, J-C (1978). De la syntaxe à l'interprétation (Quantités, insultes, exclamations). Paris : Le Seuil

Molinier, Ch. (1990). Une classification des adverbes en -ment, in Nolke, H. (éd.), Langue française 88, Classification des adverbes, 28- 40

Molinier , Ch. et Levrier, F. (1999). Grammaire des adverbes, Description des formes en ment, Genève, Librairie DROZ S.A

Montgomery L.M. (2008). La Vallée arc-enciel. Canada : Québec Amérique

Milner, J.Cl. (1978). De la syntaxe à l'interprétation: Quantité, insultes, exclamations. Paris : Le Seuil.

Muller, Cl. (1996). La subordination en français. Paris : Armand-Colin

Natter, R. (2008). A l'ombre d'un gardien. Lille : Dynavie-création

Netchvolodow A., L'empereur Nicolas II et les Juifs, Essais sue la révolution russe dans les rapports avec l'activité universelle $d u$ judaïsme contemporain. Paris: Etienne CHIRON

Noailly, M. (1999). L'adjectif en français. Paris : Ophrys

[ralphgull] (s.d.) Repéré le 06/03/2016 à consulté le 06/03/2016 à http://ralphgull.over-

blog.com/2016/02/wheels-offortune.html

Paul, A. (2008). Oumrân et les Esséniens: L'éclatement d'un dogme. Paris : Cerf

Paquis, Cl. (2012). Fascisme et boule de gomme. Lulu.com

Prêtre, I. (1998). Ce Dieu qui nous dérange. Paris : Médiaspaul
Proust, M. (1922). A la Recherche du temps perdu.16. La Prisonnière. Paris : Editions de la Nouvelle Revue Française

(1922). A la Recherche du temps perdu.11. Sodom et Gomorrhe. Paris : Editions de la Nouvelle Revue Française

Riegel, M., Pellat, J-C, et Rioul, R. (1994). Grammaire méthodique du français. Paris: PUF.

Roigoon, F. (2011). La vengeance de MarieAntoinette. Sevres: Les éditions de l'éphémère

Romero, Cl. (2001), L'intensité en français contemporain, Analyse sémantique et pragmatique, Thèse de doctorat, Université de Paris 8.

Samain, A. (1914). Le chariot d'or. - Symphonie hérö̈que. Paris : Mercure de France

Salvayre, L. (1995). La Puissance des mouches. Paris : Le Seuil

Simard, L. (2004). La promesse, la route de l'exode. Outremont : Libre expression

Simonin, A. (1953). Touchez pas au grisbi! Paris : Gallimard

Tellez, B. (2008), Les Rescapés du Tage. Paris : Publibook

[Trésor de la langue française informatisé]. (s.d.). Repéré à http://atilf.atilf.fr//tlf.htm

Vivas J. (2006). La quête du Saint Graal et la mort d'Arthur, Grenoble : ELLUG

Wagner, R.L. et Pinchon, J. (1962). La grammaire $d u$ français classique et moderne. Paris : Hachette

Whittaker, S. (2002). La notion de gradation, Applications aux adjectifs. Berne, Berlin, Bruxelles, Frankfurt/M., New York, Oxford, Wien: Peter Lang. 